\title{
VR Based Movie Watching Method by Reproduction of Spatial Sensation
}

\author{
Kunihiro Nishimura $^{1}$, Aoi Ito $^{2}$, Tomohiro Tanikawa ${ }^{1}$, \\ and Michitaka Hirose ${ }^{1}$ \\ ${ }^{1}$ Graduate School of Information Science and Technology, The Univeristy of Tokyo \\ ${ }^{2}$ Graduate School of Interdisciplinary Information Studies, The University of Tokyo \\ 7-3-1, Hongo, Bunkyo-ku, 113-8656, Tokyo, Japan \\ \{kuni, aoi, tani, hirose\}cyber.t.u-tokyo.ac.jp
}

\begin{abstract}
A conventional movie watching method is to view movies in front of a large screen such as theaters. Conventional presenting images in fixed position have a problem that it is easy for audiences to lose their spatial sensation of existing movies. In this paper, we propose a novel movie watching method in order to improve presence in existing media contents using virtual reality technology. We assumed when frames are presented with shooting angle based on audiences' looking position, their presence will be much higher. To represent the camera-shooting angle, we used a optical flow method. We proposed a movie watching viewing method based on the reconstructed camera shooting angle which is presented with a moving projector or a wall screen. We thought that our method made it possible to reconstruct lost spatial in movies.
\end{abstract}

Keywords: Presence, Camera Work, Roaming Images, a Moving Projector, and Spatial Sensation.

\section{Introduction}

Recently, displays and projectors have been improved recently. It leads users to buy projectors for their personal use in their home, for example, making a home theater. In addition, the media of movies are increased such as DVD and Blue-ray. We can enjoy high resolution and high quality movies with both projectors or displays and digital medias.

Displays and projectors are generally used to present images, and they come to enable users to feel presence. There are several ways to improve presence. Researches improved presence are followings; expression of immersion [1], image moseying for generating a large and high resolution images by integrating multiple images [2][3][4], and construction of 3D space from multiple images or movies[5][6]. Researches that relate to reconstruct 3D geometry using an optical flow have been conducted for many years [7][8]. Study about 3D reconstruction calculated by optical flow from pre correction camera [9][10].

In movie theatre, wide view screen and high resolution displays are introduced. With using a still projector that are used in general, shooting loses spatial sensation because presenting images cannot be moved. It leads to lose the spatial sensation that was taken at the movie shooting. 

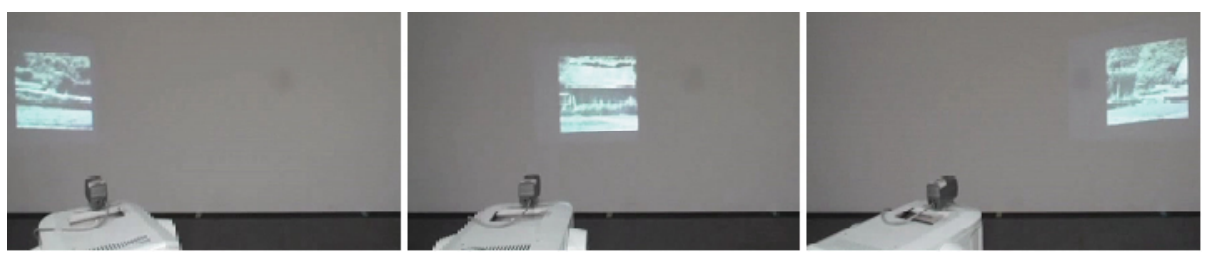

Fig. 1. Proposed Method using a Moving Projector

In this paper, we propose a novel VR based movie-watching method of improving presence of existing media contents. Our proposal method makes it possible to reconstruct lost spatial sensation in the movie. By presenting frames with shooting angle based on audiences' looking position, lost spatial sensation can be reconstructed and audiences can feel higher presence. We reconstruct camera work and present images by roaming images with a moving projector based on camera work calculated from movies (Fig.1). We also present movies not only current frames but also past frames using a wall type display. The frames are presented in a wide view display based on the spatial angle.

\section{Spatial Sensation and Camera Work in a Movie}

\subsection{Spatial Sensation}

In this paper, spatial sensation is defined as extensity. That is to say, recognizing spatial sensation means to grasp the position of objects or situation with high accuracy. We consider recognizing spatial sensation make us feel high presence. However, the existing methods of movie appreciate are difficult to express accurate space in movies because the position of presenting images are fixed. Thus, we reconstruct the space of movie for grasping the position of objects or situation in movie with accuracy.

Our method helps to construct space in movie and to grasp spatial sensation intuitively. In a movie theatre, we can see a large wide view screen, which enable us to fell presence. However, we know presence consists of not only a large wide view but also a spatial sensation in a real world. We can feel directions of objects, position of objects, and relationship among them. We often remember the spatial relationship among objects. For example, there is a kiosk shop next to a station and there is a police station in front of the kiosk shop. We remember the position and the direction of such objects. We focused on this kind of sensation in order to improve presence. When we watch movies in a conventional movie watching methods, we have to reconstruct object positions in our mind. We have to remember the previous frames that present one object and have to grasp the camera work in order to know the relationship between the object and a new object. That is, we reconstruct our spatial sensation by ourselves.

\subsection{Camera Work}

Many movies with complex camera work are existed. However, according to "Grammar of the film Language" written by D.Arijon, the movements of camera itself are 
only panning (to rotate the camera angle with camera's position fixed), traveling (to move camera position) and zooming (to change focal distance of a lens) [11]. We consider the effective method of presenting images for every movement of camera. In this paper, we focus on panning and constructed the system applicable only for panning.

\section{System}

In our system, we calculated shooting camera angle from a movie to reconstruct spatial sensation that was lost by shooting. To present images based on estimated camera work can comprehend intuitively space in a movie.

Fig. 2 shows a concept sketch of our system. Our system is composed of two parts; the process of calculating camera angle and the process of presenting images (Fig.3). In the process of calculating camera angle, we computed the camera angles when the movie was shot from a camera. In the process of presenting images, we projected movie frames at the position synchronized with calculated camera angles.

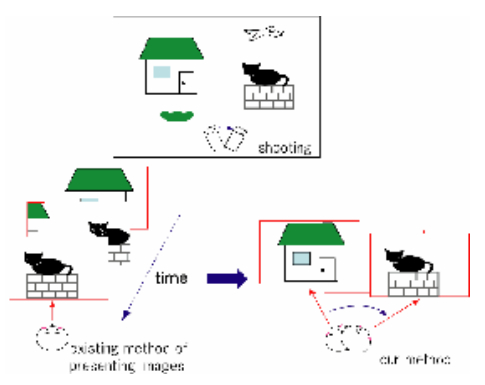

Fig. 2. Concept Image of Proposed Method

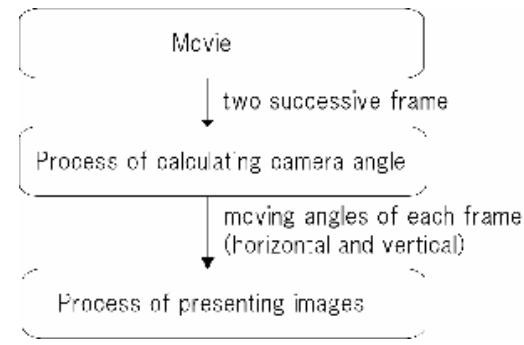

Fig. 3. System Overview

\subsection{Process of Calculating Camera Angle}

In this paper, spatial sensation is defined as extensity. That is to say, recognizing spatial sensation means to grasp the position of objects or situation with high accuracy. We consider recognizing spatial sensation make us feel high presence. However, the existing methods of movie appreciate In order to apply also to existing movies, we computed camera angles not by sensors such as gyroscope and accelerometer, but by optical flow (calculated velocity field between successive.) Because current movies did not taken with such sensors. Using optical flow enables to calculate differential angle between two successive frames.

We used OpenCV library that Intel Corporation have been developed and opened for image processing when we calculate optical flow between images. Optical flow has several methods--gradient method, Block-Matching method and so on. We use iterative Lucas-Kanade method in pyramids [12]. Lucas-Kanade method is one of the local gradient methods that is said to be one of the best algorithm for this usage because of high processing speed and high level of confidence [13]. This method 

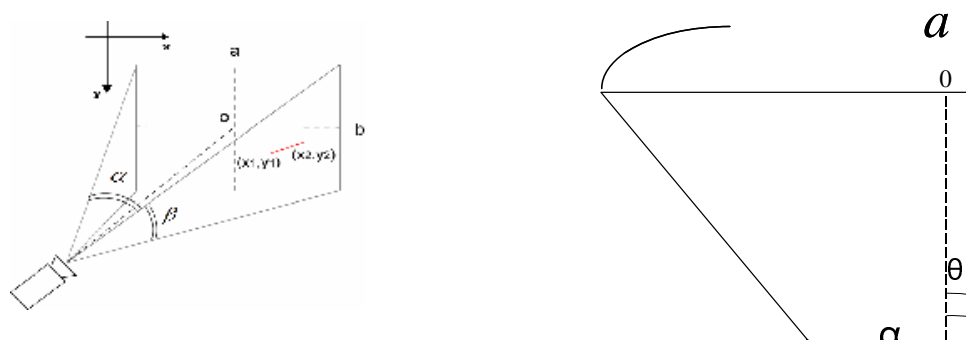

Fig. 4. The relationship between a camera and an image

can give us accurate movement of each feature point. We divide a movement into horizontal element and vertical element and respectively calculate differential angles by the angle of view of a camera and the size of a frame image. Fig 4 shows the relationship between a viewpoint and a frame.

Coordinate $x_{l}$, which represents the position of feature point of former frame and coordinate $x_{2}$, which represents that of latter frame can be calculated by using optical flow. Suppose that the angle of view of a camera $(\alpha)$ and the size of a frame image (a) are known. The differential angle $(\theta)$ is difference between $\theta_{l}$, which is the angle between coordinate $x_{1}$ and the center of the image, and $\theta_{2}$, which is the angle between coordinate $x_{2}$ and the center of the image.

$$
\begin{gathered}
\theta_{1}=\arctan \left(\frac{x_{1}-a / 2}{l}\right) \\
l=\frac{a / 2}{\tan (\alpha / 2)}
\end{gathered}
$$

$l$, which is the length from the center of the image to the camera is calculated by (2).

$$
\theta_{1}=\arctan \left(\frac{\frac{x_{1}-a / 2}{a / 2}}{\tan (\alpha / 2)}\right)
$$

$\theta_{1}$ is calculated by (3). $\theta_{2}$ can also be calculated by the same process. $\theta$ is difference of $\theta_{1}$ and $\theta 2$.

However, when we calculate camera angles only by a movie, we cannot know the angle of view of a movie. So, we calculate virtual camera angles using parameters of the projector. We use the angle of projection ( $\alpha$ ) and the size of projection (a) and calculate camera angles.

In our method, optical flow is calculated after extracting many feature points. There are several optical flows, so we average them as the difference angle between two frames. We calculate the difference angles between every two frames. 

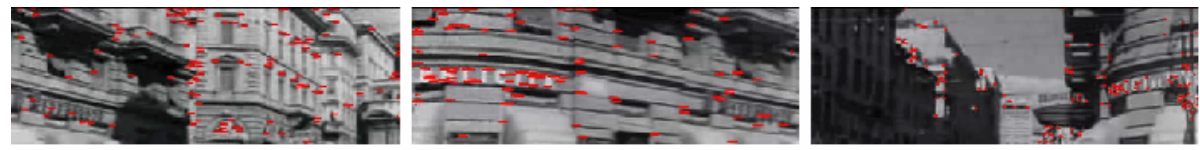

time

Fig. 5. Optical flows in the upper part of frames

However, when we don't account for moving objects, we can't calculate accurately the different angles because the optical flows of moving objects may be noises. Therefore, we focus only on stopped objects and calculate optical flows only from them.

For example, a movie scene sometimes does not have moving objects in the upper half of the frames. We should use the upper half of the frames for calculating the optical flow. We use equation (1)-(3) for calculating camera's relative moving angle. Fig 5 shows the picture of calculating the optical flow of the upper half of the frames.

\subsection{Process of Presenting Images}

In the process of presenting images, we used a moving projector "Active Vision" produced by TOSHIBA LIGHTING \& TECHNOLOGY CORPORATION. The moving projector can change the direction of projection either horizontally or vertically. We reconstructed the shooting camera angles by directing the moving projector to the angle calculated by the process of calculating camera angle. The moving projector can move at most 80 degrees/sec. The size of presenting images depends on the size and the aspect ratio of the projector.

We need to revise the projecting images because the angle between the plane of projection and the line of projection is changed by moving the projector. We project the images revised by changing the shape of images, not by changing the parameter of the projector. Fig. 1 shows the appearance of projecting images.

\subsection{Process of Presenting Images with Frame Logs}

In the process of presenting images, we also used a wall type screen. When we used a moving projector, we could know the spatiality because we could know the exactly presented angle. It was easy for us to grasp relationships among objects such as a buildings and road. However, a moving projector has a limitation. It can present images on a restricted area. We could not see the previous frames. We should remember the frames when presented images are moving. Thus we propose a new combine method in order to present both spatiality and frame histories. We used a wall type screen $(9 \mathrm{~m}$ wide $\times 2.7 \mathrm{~m}$ height). The past frames are left on the wall screen. Thus audiences can easy to grasp the spatiality when a movie moves a lot.

\subsection{Implementation Results}

We applied our method to various movies. Fig. 6 shows the result of an applied movie. Our method helps to construct space in movie and to grasp spatial sensation intuitively. 

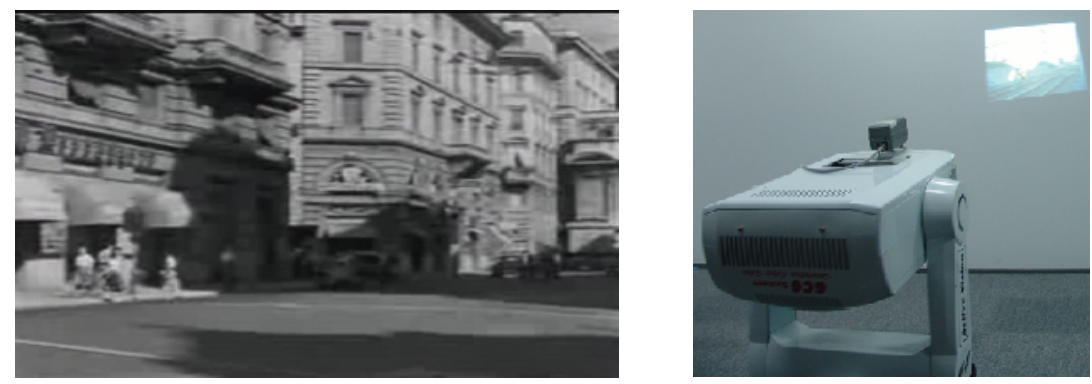

Fig. 6. (left) The result of Roman Holiday[14] (right) Appearance of presenting images using moving projector

\section{Evaluations}

\subsection{Evaluation of the Process of Calculation Camera Angle}

We applied In order to meet our proposal purpose; we should calculate the camerashooting angle precisely. In this section, we evaluated the process of calculating camera angle using the optical flow methods. The way of evaluation is by comparison between the result of calculation and the real movement angle. We calculated the camera angle when we took a movie with a panning. We compared the moving angle between observed and calculated angles.

We examined about seven movies that are different of movement angle at three different places. Table.1 shows the result of this examination. The ratio of the difference of the movement angle calculated by the theory and our system to theoretical movement angle is $7.5 \%$ on an average. This is sufficient accuracy to our study and this result implied that the process of calculating camera angle by optical flow revealed the effectiveness.

Table 1. Comparison between the translated angle calculated from theoretical and this system

\begin{tabular}{cccc}
\hline $\begin{array}{c}\text { The angle of } \\
\text { theory } \\
\text { (degree) }\end{array}$ & $\begin{array}{c}\text { The angle of our } \\
\text { system } \\
\text { (degree) }\end{array}$ & $\begin{array}{c}\text { error } \\
\text { (degree) }\end{array}$ & $\begin{array}{c}\text { The rate of } \\
\text { error } \\
\text { (percent) }\end{array}$ \\
\hline 38.7 & 35.1 & 3.6 & 9.3 \\
29.7 & 27.3 & 2.4 & 8.1 \\
16.0 & 14.3 & 1.7 & 10.6 \\
21.3 & 21.8 & -0.5 & 2.3 \\
28.8 & 27.4 & 1.4 & 4.9 \\
37.4 & 32.2 & 5.2 & 13.9 \\
29.0 & 28.0 & 1.0 & 3.4 \\
\hline
\end{tabular}




\subsection{Experiment of Grasping the Objects' Position by Reconstruction Shooting Camera Angle}

We as a pilot experiment, we examined on six subjects whether presenting images by a moving projector enables users to grasp accurately the position of objects in a movie by comparing two projection methods--projecting position is fixed and move to reconstruct the shooting camera angles.

Method of Experiment. First, we shoot three objects in a room by rotating camera horizontally. Second, subjects observe in two methods--projecting position is fixed and move to reconstruct the shooting camera angles calculated by optical flows. Third, the subjects draw the position of three objects in the movie and answer several questions. The subjects observe two movies--first, by the method of the fixed position of presenting images (non-moving) and second, by the method of the roaming images (moving). We categorized six subjects into two groups (group I, II) and change the order of presenting two movies (movie A, B), where the position of the three objects are different. Fig.7 shows the appearance of this experiment.

Result and examination. Fig.7 shows the difference of actual positions of the objects and written positions by a subject.

Table 2 shows the group I subjects experimental results, which is the angle between the position of objects in the real world and the written position of the objects by the subject. Table 3 shows the group II subjects experimental results for the sample. Fig 8 shows the graph of the same result of Table 2 and Table 3. Fig. 8 shows
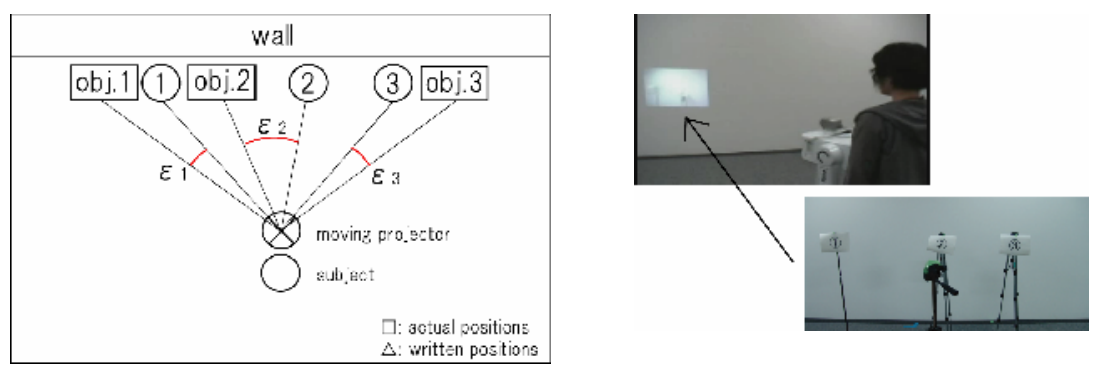

Fig. 7. The relationship between a camera and an image

Table 2. The angles of actual positions and written positions by group II

\begin{tabular}{c|ccc|ccc}
\hline Group I & \multicolumn{3}{|c|}{ Non-moving (movie A) } & \multicolumn{3}{c}{ Moving (movie B) } \\
subject & $\varepsilon_{1}$ & $\varepsilon_{2}$ & $\varepsilon_{3}$ & $\varepsilon_{1}$ & $\varepsilon_{2}$ & $\varepsilon_{3}$ \\
\hline 1 & 7.0 & 5.0 & -30.0 & -10.0 & -10.0 & -9.0 \\
2 & -14.0 & -17.5 & -20.0 & 16.0 & 5.0 & 20.0 \\
3 & 7.0 & 6.0 & -31.0 & 10.0 & 5.0 & -3.0 \\
\hline Average & 9.3 & 9.5 & 27.0 & 12.0 & 6.7 & 10.7 \\
(abs) & & & & & & \\
average & \multicolumn{3}{|c}{15.3} & & & \\
\hline
\end{tabular}

Table 3. The angles of actual positions and written positions by group II

\begin{tabular}{c|ccc|ccc}
\hline Group II & \multicolumn{3}{|c|}{ Non-moving (movie B) } & \multicolumn{3}{c}{ Moving (movie A) } \\
subject & $\varepsilon_{1}$ & $\varepsilon_{2}$ & $\varepsilon_{3}$ & $\varepsilon_{1}$ & $\varepsilon_{2}$ & $\varepsilon_{3}$ \\
\hline 4 & 15.0 & 3.0 & -8.0 & 10.0 & 10.0 & -8.0 \\
5 & 10.0 & 42.0 & 9.0 & 14.0 & 16.5 & 3.5 \\
6 & 11.0 & 11.0 & 20.0 & 13.0 & 16.5 & -12.0 \\
\hline Average & 12.0 & 18.7 & 12.3 & 12.3 & 14.3 & 7.8 \\
(abs) & & & & & & \\
average & & 14.3 & & \multicolumn{4}{|c}{10.6} \\
\hline
\end{tabular}




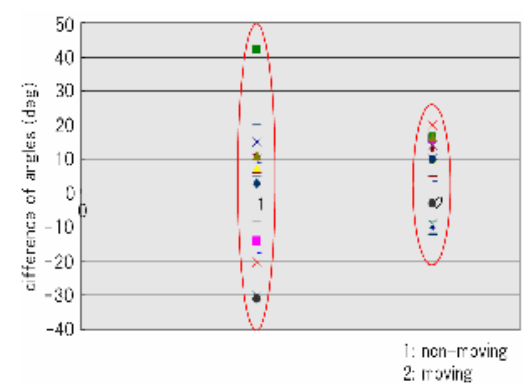

Fig. 8. The angles of actual positions and written positions by all subjects each case of nonmoving and moving

Table 4. Answer of the question "Which task is easy for you to grasp the object position"

\begin{tabular}{c|c|c|c}
\hline groupl & answer & groupll & answer \\
\hline Subject 1 & 5 & Subject 4 & 5 \\
Subject 2 & 4 & Subject 5 & 3 \\
Subject 3 & 5 & Subject 6 & 5 \\
\hline
\end{tabular}

the angles of actual positions of objects and written positions by subjects (both group I and II) in each case of non-moving and moving. Many subjects can grasp more accurately the position of the objects in the case of moving than in the case of nonmoving since the more the difference angle between actual and written objects close to 0 degree, the more the subjects grasp the positions of the objects.

We conducted questionnaires for subjects. Table 4 shows the result of the question; "Which task is easy for you to grasp the object position?" A result of questions suggests that the method of reconstructing the shooting camera angles got high valuation from most subjects. This means that subjects can grasp the positions of objects easily. We can suggest that roaming images is effective to improve presence and grasp the space precisely in the movie.

We shoot three objects in a room by rotating camera horizontally. Second, subjects observe in two methods--projecting position is fixed.

\subsection{Experiment with Existing Movies}

We compared two method applied for the existing movie. We employed six subjects and conducted the experiment that can compare subjects' feelings under two conditions; present images at a fixed position and presenting images based on the reconstructed camera work. Subjects were asked to answer several questions. The positions of the screen, the moving projector, and subjects were the same as the previous pilot experiment. We categorized six subjects into two groups (group I, II), used two different movies (movie A, B), and changed the order of presenting method as well as the pilot experiment. In group I, subjects observed the both movies with the method of the fixed position first and then observed the movies with our proposal method. 
In group II subjects observed the both movies with our method first and then observed the movies with the method of the fixed position. We presented the movie a first in both methods. Subjects were asked to answer the questions after observing each movie four times.

We prepared five questions and subjects replied them by choosing five answers; strongly disagree, disagree, neutral, agree, and strongly agree.

- Could you grasp what occurred in the movie?

- Could you grasp the space in the movie?

- Did you feel the space is wide?

- Did you feel you observed in the space?

- Were you interested?

Moreover, subjects asked to answer the most impression point.

In each movies, five subjects thought it was possible to grasp the spaces in the movies using our method, thus it suggested that our method was effective to reconstruct the lost spatial sensation. In addition, five subjects in the case of movie A and four subjects in the case of movie B felt that they were there in our method. In each movie, one subject who answered "disagree" answered the same score or higher score in our method than the other method. Therefore, it was suggested that our method could improve presence.

In group I (with the method of the fixed position of presenting images first), the impressed objects in the movies were same in the case of our method and in the case of the method of the fixed position of presenting images, but in group II (with our method first), the impressed objects in the movies were different by two methods. Additionally, most subjects of group I were impressed by concrete objects in the movie, but most subjects of group II replied sensational answers. These results implied that subjects felt differently between the method of the fixed position of presenting images and the method of the moved position of presenting images.

\section{Conclusion}

In this paper, we proposed the novel presentation method of improving presence for existing media contents such as movies. The method includes a reconstruction of the lost spatial sensation in movies and presentation of images at the right direction which angles are calculated by optical flow. This method enables us to improve our sense of spatiality and presence. A pilot experiment indicated that present images based on the reconstructing camera work makes us more accurately grasp the space that are expressed in the movie than presenting images at a fixed position. In addition, an application for existing movies revealed that our method was effective in reconstructing the lost spatial sensation in movies and improving presence.

Acknowledgments. A part of this study is supported by JST CREST "Technology to Create Digital Public Art". 


\section{References}

1. Hirose, M.: CABIN-A multiscreen display for computer experiments. In: 1997 Int. Conf. on Virtual Systems and MultiMedia, p. 78 (1997)

2. Chenm, S.E.: QickTime VR - an image-based approach to virtual environment navigation. In: Proc. SIGGRAPH 1995, pp. 29-38 (1995)

3. Sezliski, R., Shum, H.Y.: Creating full view panoramic image mosaics and environment maps. In: Proc. SIGGRAPH 1997, pp. 251-258 (1997)

4. DiVerdi, S., Wither, J., Hollerei, T.: Envisor: Online Environment Map Construction for Mixed Reality. In: IEEE Virtual Reality 2008, pp. 19-26 (2008)

5. Hoiem, D., Efros, A.A., Hebert, M.: Automatic photo pop-up. In: Proc. SIGGRAPH 2005, pp. 577-584 (2005)

6. Van de Hengel, A., Dick, A., Thormahlen, T., Ward, B., Torr, P.H.S.: VideoTrace: Rapid interactive scene modelling from video. In: Proc. SIGGRAPH 2007, p. 86 (2007)

7. Longuet-Higgis, H.C., Prazdny, K.: The interpretation of a moving retinal image. In: Proc. Roy. Soc. Lond., vol. B208, pp. 385-397 (1980)

8. Maybank, S.J.: The angular velocity associated with a optical flow field arising from motion through a rigid environment. Proc. Roy. Soc. Lond. A401, 317-326 (1985)

9. Brooks, M.J., Chojnacki, W., Baumera, L.: Determining the egomotion of an uncalibrated camera from instantaneous optical flow. J. Opt. Soc. Am., A 14-10, 2670-2677 (1997)

10. Viéville, T., Faugeras, O.D.: The first order expansion of motion equations in the uncalibrated case. Computer Vision Image Understanding 64(1), 128-146 (1996)

11. Arijon, D.: Grammar of the Film Language. Focal Press, London (1976)

12. Lucas, B., Kanade, T.: An Interative Image Registration Technique with an Application to Stereo Vision. In: Proc. The 7th International Joint Conf. on Artificial Intelligence, pp. 674-679 (1981)

13. Baron, J., Fleet, D., Beauchemin, S.: Performance of optical flow techniques. Int. J. Computer Vision 12(1), 43-77 (1994)

14. Wyler, W.: Roman Holiday (1953) 